\title{
RESSONÂNCIA MAGNÉTICA HEPÁTICA EM PUÉRPERAS ESTÁVEIS COM SÍNDROME HELLP
}

\author{
Ana Rita Marinho Ribeiro Carvalho, Melania Maria Ramos de Amorim*, Leila Katz, Alex Sandro Rolland de Souza, Aleksana Regina Viana Dutra
} Santos, Ana luíza Medeiros Vasconcelos de Lima

Trabalho realizado no Centro de Atenção à Mulher - Unidade de Terapia Intensiva Obstétrica do Instituto Materno Infantil Prof. Fernando Figueira, Recife, PE.

${ }^{*}$ Correspondência:

Rua Neusa Borborema, 300

Bairro Santo Antônio

Recife - PE

CEP 58103-313

Tel: (83) 8822-1514

melamorim@uol.com.br

\begin{abstract}
RESUMO
Oвjetivos. Descrever os achados hepáticos na ressonância magnética em puérperas estáveis com síndrome HELLP.

Métodos. Realizou-se um estudo descritivo, do tipo série de casos, envolvendo 40 puérperas internadas na UTI obstétrica do Instituto Materno Infantil Prof. Fernando Figueira (IMIP), com diagnóstico de síndrome HELLP completa (presentes todas as alterações laboratoriais) e incompleta (uma ou mais alterações laboratoriais, porém sem todos os critérios diagnósticos) no período de agosto de 2005 a julho de 2006.
\end{abstract}

Resultados. A idade média foi de 26,8 \pm 6,4 anos, com idade gestacional média no parto de 34 semanas. A ressonância magnética foi realizada entre oito e 96 horas depois do diagnóstico de síndrome HELLP (média de $56+31$ horas). 0 achado mais freqüente foi ascite em $20 \%(n=8)$, seguindo-se derrame pleural $(17,5 \%)$ e esteatose hepática $(7,5 \%)$. A intensidade de sinal periportal foi normal em todos os casos e não se observaram casos de isquemia/infarto hepático ou de hematoma parenquimatoso ou subcapsular.

Conclusão. Os achados da ressonância magnética pós-parto em puérperas estáveis com síndrome HELLP foram inespecíficos e, na presente série, não foram encontradas lesões importantes como hematoma parenquimatoso ou subcapsular, representando risco de vida para a paciente. Os resultados encontrados não corroboram a utilização desse exame de rotina para o seguimento de pacientes com síndrome HELLP.

UniteRMOS: Síndrome HELLP. Pré-eclâmpsia. Imagem por ressonância magnética. Gravidez de alto risco.

\section{INTRODUÇÃO}

A síndrome HELLP $(H=$ hemolysis, EL = elevated liverfunction, $L P$ = low platelet count), descrita por Weinstein, em 1982, caracteriza-se por hemólise, aumento de enzimas hepáticas e plaquetopenia!. Freqüentemente encontra-se associada à pré-eclâmpsia e eclâmpsia e cursa com disfunção hepática durante a gestação².

Dentre os casos de pré-eclâmpsia, sua freqüência é variável, provavelmente devido às diferentes formas clínicas das síndromes hipertensivas e aos diferentes critérios diagnósticos utilizados ${ }^{2,3}$. Em nosso meio encontrou-se uma freqüência de $46 \%$ de síndrome HELLP entre pacientes admitidas em uma UTI obstétrica por distúrbios hipertensivos ${ }^{4}$.

O diagnóstico precoce é laboratorial e deve ser pesquisado de maneira sistemática nas mulheres com pré-eclâmpsia e eclâmpsia. Sibai et al., em 1990, propuseram uma sistematização dos padrões laboratoriais e bioquímicos para o diagnóstico dessa síndrome, como hemólise (esfregaço de sangue periférico com esquizócitos, bilirrubina total > 1,2 mg/dl e desidrogenase láctica - DHL > $600 \mathrm{U} / \mathrm{L}$ ), elevação de enzimas hepáticas (aspartato aminotranferase - AST > $70 \mathrm{U} / \mathrm{L}$ e $\mathrm{DHL}>600 \mathrm{U} /$ ) e plaquetopenia (plaquetas $\left.<100.000 / \mathrm{mm}^{3}\right)^{2}$.

Diversas críticas em relaçãa ao rigor dos critérios diagnósticos foram feitas ${ }^{5-8}$, e muitos autores passaram a utilizar o conceito de síndrome HELLP parcial ou incompleta, ou seja, pacientes com uma ou mais, porém não todas as alterações laboratoriais consideradas como critérios diagnósticos da síndrome 9 . Considerando que a doença é progressiva e que mulheres que apresentam alguns critérios diagnósticos podem progredir para o quadro completo quando não interrompida a gestação, o diagnóstico de síndrome HELLP incompleta pode contribuir para a decisão terapêutica?. Alguns autores sugerem aumento da morbimortalidade materna em pacientes com a síndrome incompleta quando comparadas com as pacientes com pré-eclâmpsia sem síndrome HELLP, mesmo que em menor grau que as pacientes com o quadro completo $^{10}$. Além disso, o diagnóstico de quadros intermediários foi reiterado quando foram descritos quadros de ruptura hepática em pacientes com a classe 3 de Martin".

A síndrome HELLP representa um quadro grave dentre as doenças hepáticas que acometem a gravidez, por oferecer alto risco de mortalidade materna ${ }^{2-5,16}$ e perinatal ${ }^{12-14}$. A mortalidade materna na síndrome HELLP é extremamente variável, oscilando entre 1\% e 24\% dos casos, sendo os óbitos decorrentes em $80 \%$ das vezes de complicações do sistema nervoso central. As demais causas de mortalidade são atribuídas às complicações hepáticas, como hematoma subcapsular e ruptura, infarto e insuficiência hepática fulminante, além de embolismo pulmonar, encefalopatia hipóxica, coagulação intravascular disseminada, falência renal aguda ${ }^{2-5,16}$.

Pelo fato de o acometimento hepático ser um evento de grande importância, é fundamental investigar métodos diagnósticos que possam permitir sua detecção mais precoce. Assim, os exames de imagens 
são fundamentais para essas finalidades ${ }^{15-19}$. Na literatura, revisando-se os bancos de dados MEDLINE, EMBASE, LILACS, SCIELO e SCOPUS, encontram-se relatos de casos e séries de casos abordando principalmente as complicações da síndrome. Pouco se sabe sobre as alterações de imagens das pacientes estáveis.

A ressonância magnética (RM) é um excelente método para avaliação hepática. Os achados da síndrome HELLP, como anormalidades periportais intra-hepáticas, infiltração gordurosa no figado, infarto hepático e hemorragia hepática espontânea estão bem descritos na literatura ${ }^{17,19,20}$, mas não está definido quando o exame deve ser realizado e qual a sua importância na síndrome HELLP.

Barton e Sibai, em 1996, avaliando o perfil de imagem de 34 pacientes com síndrome HELLP completa, observaram em $47 \%$ alterações hepáticas. Entretanto, a única alteração laboratorial que apresentou correlação com os exames de imagem foi a trombocitopenia, observando-se que de 13 pacientes com plaquetas abaixo de 20.000/ $\mathrm{mm}^{3}, 10$ apresentavam alterações hepáticas nos exames de imagem. Os autores recomendam os exames de imagem nas pacientes com suspeita clínica de hematoma hepático e naquelas com plaquetopenia $\left(<20.000 / \mathrm{mm}^{3}\right)^{15}$.

O presente estudo foi realizado para descrever as alterações hepáticas na ressonância magnética em puérperas com síndrome HELLP clinicamente estáveis, tentando discriminar se achados anormais associam-se à piora do prognóstico.

\section{Métodos}

Foi realizado um estudo descritivo, tipo série de casos, na Unidade de Terapia Intensiva (UTI) Obstétrica do Instituto Materno Infantil Professor Fernando Figueira (IMIP), Recife, Pernambuco, Brasil, no período entre agosto de 2005 e julho de 2006. A rotina da instituição é que todos os casos de pré-eclâmpsia grave e eclâmpsia sejam internados neste setor para monitorização da paciente e administração intravenosa do sulfato de magnésio 6 .

Obteve-se uma amostra de conveniência, incluindo-se 40 puérperas com diagnóstico de síndrome HELLP, internadas na UTI Obstétrica do IMIP após o término da administração do sulfato de magnésio e que se submeteram à RM com, no máximo, sete dias pós-parto. Excluíram-se as pacientes com instabilidade clínica, que impedisse a locomoção para realização do exame, qualquer condição impossibilitando a colaboração com o exame e/ou a decisão sobre a participação no estudo, presença de hepatopatias prévias conhecidas e contra-indicações à realização da ressonância magnética, usuárias crônicas de corticosteróides e antiinflamatórios não hormonais, portadoras de vasculites, lupus, púrpuras, estados de hipercoagulabilidade, outras doenças imunológicas e antecedente recente de trauma abdominal.

As síndromes hipertensivas da gestação foram definidas segundo os critérios do National High Blood Pressure Education Program Working Group on High Blood Pressure in Pregnancy (20002) . Asíndrome HELLP foi caracterizada como a presença de hemólise (esfregaço de sangue periférico com esquizócitos, bilirrubina total $>1,2 \mathrm{mg} / \mathrm{dl}$ e desidrogenase láctica - DHL > $600 \mathrm{U} / \mathrm{L}$ ), elevação de enzimas hepáticas (aspartato aminotranferase - AST > $70 \mathrm{U} / \mathrm{L}$ e DHL > $600 \mathrm{U} / \mathrm{l}$ ) e plaquetopenia (plaquetas $\left.<100.000 / \mathrm{mm}^{3}\right)^{2,21}$.
Características sócio-demográficas foram colhidas, além de variáveis da gestação, do parto e do puerpério. Os principais parâmetros clínicos maternos também foram estudados, como: a época e o tempo do diagnóstico da síndrome HELLP; a classificação da síndrome HELLP, em "completa" (todos os critérios presentes) e "incompleta" (um ou mais dos critérios diagnósticos presentes, porém não todas as alterações laboratoriais); ; a classificação quanto à gravidade, em síndrome HELLP clínica, (quando havia sintomas) e síndrome HELLP laboratorial (diagnóstico laboratorial sem qualquer manifestação clínica associada); classificação da síndrome hipertensiva, como pré-eclâmpsia pura, superposta ou eclâmpsia; e a pressão arterial sistólica e diastólica.

Os exames laboratoriais foram coletados e analisados na admissão das pacientes na UTI obstétrica. Os parâmetros estudados por RM foram: dimensões hepáticas, maior extensão longitudinal dos lobos direito (até $13 \mathrm{~cm}$ ) e esquerdo (até $10 \mathrm{~cm}$ ), realizadas no plano coronal; intensidade de sinal hepático; esteatose hepática; intensidade de sinal e impregnação periportal; impregnação do parênquima hepático; hematoma hepático intraparenquimatoso; hematoma subcapsular; lesão hepática focal; presença de ascite e derrame pleural; e outros achados, caso presentes.

Os exames foram realizados em uma clínica privada da cidade do Recife, em aparelho General Eletric (GE) SIGMA I,5 T utilizando-se bobina de sinergia (Torso). As pacientes eram transportadas em ambulância do IMIP acompanhadas de auxiliar de enfermagem. O tempo de duração do exame era de aproximadamente 30 minutos, permanecendo a paciente em decúbito dorsal no interior do magneto. A administração intravenosa do contraste Gadolínio era realizada através de veia periférica em membro superior ou de veia central.

A RM foi realizada com a paciente respirando espontaneamente e em um período de apnéia de aproximadamente 20 segundos. A seqüência TI FSPGR com saturação de gordura foi realizada antes do contraste e nas fases arterial (30 segundos), portal (50 segundos) e de equilíbrio (3 minutos), as três primeiras no plano axial e a fase de equilíbrio no plano coronal. A dose do agente paramagnético realizado foi de 0, I mmol de Gadolínio por kg de peso corporal (equivalente a 0,2 $\mathrm{ml} / \mathrm{kg}$ de peso corporal). Todos os exames foram avaliados por um radiologista com experiência em RM hepática e que não tinha conhecimento das alterações clínicas ou laboratoriais das pacientes.

A análise dos dados foi realizada usando o programa Epi-Info 3.4. I, de domínio público. Foram construídas tabelas de distribuição de freqüência e calculadas as medidas de tendência central e de dispersão. O teste exato de Fisher foi utilizado para avaliar a associação entre o achado de esteatose hepática (presente ou ausente) e a classificação da síndrome HELLP (completa ou incompleta), considerando-se o nível de significância de 5\%.

O presente estudo obedeceu aos postulados da Declaração de Helsinque emendada em Hong Kong (1989), bem como às normas da resolução 196/96 do Conselho Nacional de Saúde. O estudo foi iniciado apenas após a aprovação pelo Comitê de Ética em Pesquisa do IMIP, e as pacientes somente foram incluídas caso concordassem em participar, depois da informação, assinando o Termo de Consentimento Livre e Esclarecido. 


\section{Resultados}

As pacientes se caracterizaram por idade média de 26,8 \pm 6,4 anos de idade. Quanto à escolaridade, 36 pacientes tinham instrução formal, tendo em média 7,0 \pm 3,0 anos de estudo, sendo que 17,5\% tinham menos do que quatro anos de estudo. Em relação à procedência, predominaram mulheres do interior do estado de Pernambuco, representando $60 \%$ da amostra.

Em relação às características obstétricas, houve predomínio de primigestas que representaram $45 \%$ da amostra e a idade gestacional média no parto foi de $34,0 \pm$ 4,0 semanas. Os antecedentes de distúrbio hipertensivo em gestação anterior estiveram presentes em $32,5 \%$ das pacientes. O peso ao nascer médio foi de $1.985,8 \pm 806,8$ g e a maioria das mulheres ( $85 \%$ ) foi submetida à cesariana. Quanto às condições de nascimento, predominaram os nativivos (87,5\%).

A pressão arterial sistólica variou entre 110 e $240 \mathrm{mmHg}$, enquanto a pressão arterial diastólica teve intervalo de 80 a $160 \mathrm{mmHg}$. A hipertensão sistodiastólica foi diagnosticada em 39 mulheres e sistólica isolada em uma paciente. Quanto à classificação do distúrbio hipertensivo, a pré-eclâmpsia pura foi diagnosticada em 82,5\% dos casos, a superposta em 10\% e a eclâmpsia em 7,5\% dos casos (Tabela I).

Quanto às características da síndrome HELLP, a maioria foi diagnosticada no período pré-parto (55\%), tendo sido a RM realizada entre oito e 96 horas pós-parto. A síndrome foi completa em 50\% dos casos e clinicamente definida em 60\% dos casos (Tabela I).

$\mathrm{Na}$ avaliação das manifestações clínicas, entre as 24 pacientes $(60 \%)$ que apresentaram sintomatologia, predominaram epigastralgia (42,5\%), manifestações hemorrágicas (37,5\%), náuseas e vômitos (30\%), insuficiência renal (22,5\%), hematúruia (17,5\%), oligúria ( I5\%), colúria e icterícia (7,5\%). Em uma paciente foi diagnosticado discreto hemoperitônio na cesárea.

Entre as alterações laboratoriais, as mais freqüentes foram as eritrocitárias, identificadas em $52,5 \%$ das pacientes. A concentração hemoglobínica variou entre $3 \mathrm{~g} / \mathrm{dL}$ e $14 \mathrm{~g} / \mathrm{dL}$, enquanto o hematócrito situou-se no intervalo de $10,4 \%$ e 41 , 5\%. A elevação ALT foi vista em $45 \%$ das mulheres e 52,5\% apresentaram AST aumentada. A hiperbilirrubinemia esteve presente em 12,5\% dos casos, sempre à custa da bilirrubina indireta. A DHL esteve aumentada em 17,5\% dos casos. A uremia esteve aumentada em 35\% das pacientes, o aumento da creatinina em $30 \%$ e o aumento do acido úrico em 27,5\% das pacientes. Investigando-se a proteinúria, foram identificadas $42,5 \%$ de mulheres com proteinúria acentuada $(3+)$.

Analisando-se os achados da RM, 7,5\% das pacientes apresentavam alteração da intensidade de sinal no parênquima hepático decorrente da presença de esteatose hepática difusa. A única paciente que apresentou realce hepático alterado apresentou lesão sugestiva de hemangioma hepático. A intensidade de sinal periportal foi normal em todas as pacientes e não houve caracterização de distúrbio perfusional. Em relação ao tamanho do fígado, em 87,5\% das mulheres o lobo direito estava aumentado e em $22,5 \%$ havia aumento do lobo esquerdo. Nenhuma das mulheres apresentou isquemia ou infarto hepático, hematoma intraparenquimatoso ou subcapsular (Tabela 2).

As três pacientes que apresentavam esteatose hepática difusa diagnosticada na RM encontravam-se com forma completa da
Tabela I - Características de 40 puérperas com síndrome HELLP estáveis, submetidas à ressonância magnética

\begin{tabular}{|c|c|c|}
\hline Características da Síndrome HELLP & $\mathrm{n}$ & $\%$ \\
\hline \multicolumn{3}{|l|}{ Classificacão distúrbio hipertensivo } \\
\hline Pré-eclâmpsia pura & 33 & 82,5 \\
\hline Pré-eclâmpsia superposta & 4 & 10,0 \\
\hline Eclâmpsia & 3 & 7,5 \\
\hline \multicolumn{3}{|l|}{ Tipo da hipertensão } \\
\hline Hipertensãosistodiastólica & 39 & 97,5 \\
\hline Hipertensão sistólica isolada & 1 & 2,5 \\
\hline \multicolumn{3}{|l|}{ Classificação da HELLP } \\
\hline Completa & 20 & 50,0 \\
\hline Incompleta & 20 & 50,0 \\
\hline \multicolumn{3}{|l|}{ Quanto à presença de manifestações clínicas } \\
\hline Clínica & 24 & 60,0 \\
\hline Laboratorial & 16 & 40,0 \\
\hline \multicolumn{3}{|l|}{ Época do diagnóstico } \\
\hline Pré-parto & 22 & 55,0 \\
\hline Pós-parto & 18 & 45,0 \\
\hline
\end{tabular}

Tabela 2 - Achados hepáticos da ressonância magnética em puérperas com síndrome HELLP estáveis

\begin{tabular}{lcc}
\hline Achados da ressonância magnética & $\mathbf{n}$ & $\%$ \\
\hline Intensidade sinal & & \\
$\quad$ Alterado & 3 & 7,5 \\
$\quad$ Normal & 37 & 92,5 \\
Esteatose hepática & & \\
$\quad$ Ausente & 37 & 92,5 \\
$\quad$ Dífusa & 3 & 7,5 \\
Intensidade sinal periportal normal & 40 & 100,0 \\
Realce hepático & & \\
$\quad$ Alterado & 1 & 2,5 \\
$\quad$ Normal & 39 & 97,5 \\
Lesão hepáticafocal & & \\
$\quad$ Ausente & & \\
Presente & 39 & 97,5 \\
Lobo hepático direito & 1 & 2,5 \\
$\leq 13 \mathrm{~cm}$ & & \\
$\geq 13,1 \mathrm{~cm}$ & & \\
Lobo hepático esquerdo & 5 & 2,5 \\
$\leq 10 \mathrm{~cm}$ & 35 & 77,5 \\
$\geq 10,1 \mathrm{~cm}$ & & 22,5 \\
\hline
\end{tabular}




\begin{tabular}{lccccc}
\hline \multicolumn{3}{c}{ Tabela 3 - Distribuição de freqüência das puérperas de acordo com a } \\
presença de esteatose hepática na ressonância magnética e classificação da \\
síndrome HELLP estáveis
\end{tabular}

$p$ (teste exato de Fisher) $=0,11$

síndrome HELLP, não sendo encontrada diferença estatisticamente significante $(p=0, I I)$ (Tabela 3). Ascite e derrame pleural foram caracterizados à RM, respectivamente, em oito e em sete mulheres.

\section{Discussão}

O raciocínio norteador desta pesquisa consistiu em admitir a possibilidade de que as alterações metabólicas, identificadas pela dosagem de enzimas hepáticas, se acompanhassem por alterações estruturais do fígado, constatáveis pela RM, que constitui um método de imagem não invasivo e mais sensível para detectar e caracterizar alterações parenquimatosas focais, difusas ou distúrbios perfusionais ${ }^{17}$.

Desta forma, achava-se que o dano endotelial poderia acarretar uma redução da perfusão hepática arterial, com conseqüente aparecimento de alterações no parênquima hepático. Todavia, não se identificou esse processo no presente estudo com a realização da RM. Supõe-se que as conseqüências do insulto isquêmico para o fígado ocorrem em dimensões menores que o poder de resolução da RM, apesar dessas alterações endoteliais terem sido já comprovadas em outras pesquisas $^{18}$. No entanto, pode-se perceber que esses resultados pareceram confirmar a hipótese de Weinstein (1982) de que a lesão hepática ocorre ao nível endotelial sinusoidal intimal'.

Descrevendo as características da amostra, os resultados encontram-se de acordo com a literatura ${ }^{3,6}$. Eram mulheres com idade média de 26,8 anos e procedentes em $62,5 \%$ das pacientes das cidades do interior de Pernambuco e de outros Estados. Este achado está acima do encontrado na avaliação do perfil geral dos internamentos na UTI obstétrica do IMIP4, em que se detectou uma freqüência de 47\% de pacientes procedentes de cidades do interior de Pernambuco ou outros estados. Acredita-se que esse achado pode refletir a gravidade da síndrome HELLP e a falta de recursos, pessoal ou de estrutura, na condução destas pacientes, que foram encaminhadas com maior freqüência para centros terciários na capital.

O peso médio ao nascer foi de 1.985,8 gramas, o que está de acordo com outros estudos publicados ${ }^{4,23}$. A média da idade gestacional do parto foi de 34 semanas, o que pode ser justificado pela conduta de interrupção da gestação após o diagnóstico da síndrome HELLP. O baixo peso encontra-se relacionado com a prematuridade e possivelmente com o sofrimento fetal crônico devido à hipertensão materna ${ }^{4}$. A taxa de natimortos encontrada foi de 12,5\%, compatível com a mortalidade perinatal na síndrome HELLP publicada, que varia de 5\% a $20 \%$, caracterizando a gravidade da síndrome ${ }^{14}$.
Encontrou-se uma alta freqüência de cesáreas (85\%) nas pacientes com síndrome HELLP, semelhante a outro estudo realizado no IMIP, no qual a cesariana foi realizada em cerca de dois terços dos casos. Esta elevada taxa justifica-se, em parte, pela gravidade das complicações, impondo geralmente o término da gestação em fase precoce, com condições cervicais desfavoráveis e/ou comprometimento do bemestar fetal ${ }^{4}$.

Quanto à classificação da síndrome, 50\% dos casos apresentavam a forma completa, $60 \%$ apresentavam manifestações clínicas e $40 \%$ apenas alterações laboratoriais. As manifestações clínicas, com predomínio de epigastralgia, manifestações hemorrágicas, náuseas e vômitos, confirmaram o que se vem constatando desde 1982, quando Weinstein pela primeira vez descreveu a síndrome!.

Observaram-se alterações eritrocitárias em 52,5\%, aumento da DHL em 17,5\% e hiperbilirrubinemia em apenas 12,5\%. Schwartz et al. (2000) estudando pacientes com eclâmpsia e pré-eclâmpsia, encontraram que os sintomas neurológicos estavam associados com alterações morfológicas nas hemácias e níveis elevados da DHL, achados que indicam hemólise microangiopática sugerindo dano endotelial2 ${ }^{24}$. Este dano endotelial é considerado como ponto central na fisiopatologia da eclâmpsia e pré-eclâmpsia nos múltiplos órgãos e acredita-se que esteja relacionado com toxinas endoteliais circulantes ou anticorpos contra o endotélio ${ }^{24}$. Ressalta-se que no nosso estudo nenhuma das pacientes com alterações eritrocitárias e aumento da DHL apresentou alteração na RM hepática. Desta forma, sugere-se que a circulação do sistema nervoso central é mais susceptível ao mecanismo etiopatogênico da pré-eclâmpsia. Na avaliação das enzimas hepáticas, a AST encontrouse aumentada em $45 \%$ dos casos e a ALT aumentada em 52,5\%, o que reflete o dano hepatocelular.

Em relação à avaliação renal, observou-se hiperuricemia em $27,5 \%$ dos casos, hipercreatininemia em 30\%, hipeuremia em 35\% e a proteinúria esteve presente em $77,5 \%$ das pacientes. Sabe-se que, inicialmente, o envolvimento renal na pré-eclâmpsia compromete a função tubular, refletida pela redução do clearance do acido úrico com aparecimento da hiperuricemia. Posteriormente, ocorre comprometimento da filtração glomerular e o aparecimento da proteinúria ${ }^{25}$.

Em relação aos achados da RM, uma das variáveis pesquisadas foi a maior medida longitudinal dos lobos hepáticos direito e esquerdo. Os resultados de aumento do lobo hepático direito mais acentuado que o lobo hepático esquerdo devem ser interpretados com cautela, porque não se encontrou qualquer pesquisa que fornecesse parâmetros de normalidade dessas medidas em mulheres no ciclo grávido-puerperal e, sobretudo, para portadoras de síndrome HELLP. É possível que esse aumento das dimensões do fígado possa estar relacionado ao edema intersticial devido à transudação em decorrência da lesão endotelial. Entretanto, trata-se apenas de uma descrição do resultado cujo significado poderá ser interpretado a partir de outros estudos.

O realce hepático foi normal, exceto em uma paciente que apresentou lesão hepática sólida sugestiva de hemangioma hepático, sem relação, portanto, com a síndrome HELLP. Ainda em relação ao realce, o que chamou atenção dos autores foi a ausência de distúrbios de perfusão e de sinais de infarto hepático. Como discutido, a etiopatogenia envolve dano 
endotelial e presença de vasoespasmo na circulação cerebral, o que já foi demonstrado através da angiografia digital, comprometendo artérias cerebrais de grosso e médio calibre, particularmente a artéria basilar ${ }^{26}$. Assim, acreditava-se inicialmente que poderiam ser encontradas alterações na RM relacionadas ao vasoespasmo e, conseqüentemente, redução de fluxo arterial no fígado.

Uma das hipóteses para explicar os resultados da presente pesquisa pode ter sido o exame de pacientes provenientes de um centro de referência, no qual as condutas médicas são adotadas precocemente, frustrando a alteração coagulatória da síndrome. Além do mais, como os exames de RM eram realizados em outro centro, exigindo a remoção das pacientes e os critérios de elegibilidade adotados acarretaram a exclusão dos casos mais graves, os quais poderiam ter apresentado maior freqüência de lesões evidenciáveis ao exame de ressonância. Desta forma, a lesão endotelial no fígado em pacientes com formas brandas da síndrome HELLP não determina alterações que possam ser detectáveis pela RM.

Outro aspecto a ser discutido é o tempo entre o parto e a realização da RM. Por questões logísticas, os exames eram realizados somente uma vez por semana e este fato determinou perdas de algumas pacientes, principalmente aquelas que evoluíram satisfatoriamente e tinham alta precoce da UTI. O tempo entre o parto e a realização da RM variou de oito a 96 horas, com média aproximada de 56 horas. Acredita-se que com a regressão clínica e laboratorial da síndrome, as alterações hepáticas relacionadas ao vasoespasmo sem dano estrutural do parênquima poderiam não ser caracterizadas no tempo do exame.

A infiltração gordurosa hepática esteve presente em 7,5\% dos casos, sendo descrita na síndrome HELLP em até um terço dos $\operatorname{casos}^{27}$, estando o nosso achado abaixo da média descrita na literatura. As três pacientes que apresentaram esteatose tinham síndrome HELLP completa, porém este achado não foi significativamente diferente em relação ao grupo da síndrome incompleta $(p=0,1 \mathrm{I})$. Provavelmente, a amostra da pesquisa, por ter sido pequena, também pode ter interferido neste resultado.

$\mathrm{Na}$ presença de esteatose hepática em gestante com disfunção hepática, é importante realizar diagnóstico diferencial com a esteatose hepática aguda da gestação, que é uma doença incomum, potencialmente fatal e ocorre no terceiro trimestre da gestação, havendo acúmulo microvesicular de gordura nos hepatócitos. As alterações laboratoriais podem ser semelhantes àquelas da síndrome HELLP e os exames de imagem vão diagnosticar a infiltração gordurosa do fígado. O diagnóstico é clínico e os exames de imagem são importantes para excluir outras doenças abdominais $s^{25}$.

Avaliando a intensidade de sinal periportal, observou-se que todos os exames estavam normais. Histologicamente as pacientes com eclâmpsia e pré-eclâmpsia apresentam depósitos de fibrina, hemorragia e necrose hepatocelular envolvendo áreas portais. $\mathrm{Na}$ RM pode ser observada alteração de sinal periportal, havendo relato de caso na literatura demonstrando a reversibilidade de tal achado. Este achado não tem especificidade, indicando apenas a presença de doença biliar ou hepática difusa ${ }^{19}$.

Os achados relacionados a sangramento, hematoma hepático intraparenquimatoso ou subcapsular não foram encontrados, o que era esperado em virtude dos achados normais da RM descritos e devido à exclusão do estudo das pacientes mais graves.

A síndrome HELLP é uma microangiopatia gravídica e disseminada grave, que se manifesta no segundo ou no terceiro trimestre gestacional por meio de sinais e sintomas inespecíficos e, por isso, requer vigilância intensa para que as condutas terapêuticas sejam adotadas em tempo de interromper a cascata de eventos que a caracteriza ${ }^{28}$. Devido à falta de indicativos diagnósticos atuais mais precoces que permitam preveni-la, a RM pode, no futuro, vir a constituir um método adequado para avaliação do insulto hepático na síndrome HELLP, porém os presentes resultados não apóiam a sua utilização com esta finalidade.

Até que novos estudos sejam realizados, o que se pode concluir é que pacientes com formas menos graves de síndrome HELLP no pósparto, representando a amostra do presente estudo, não requerem a realização rotineira de RM para acompanhamento ou decisão terapêutica. As indicações clássicas do exame, como na suspeita de hematoma hepático subcapsular, devem ser respeitadas, reservando-se o exame para essa hipótese diagnóstica ou os casos de recuperação mais lenta, quando exames adicionais são requeridos para o diagnóstico diferencial com outras hepatopatias.

\section{Conflito de interesse: não há}

\section{SUMMARY}

\section{MAgNetic Resonance IMAGing OF tHe LiVER IN POSTPARTUM STABLE WOMEN WITH HELLP SYNDROME}

OBJECTIVES. To describe magneticresonance (MR) findings in the liver of stable patients with HELLP syndrome in the puerpuerium.

Methods. Adescriptive study was carried out from August 2005 to July 2006, involving a series of 40 postpartum patients admitted to an obstetric intensive therapy unit in IMIP (Instituto Materno Infantil Prof. Fernando Figueira) with diagnosis of HELLP syndrome (complete and partial). Complete HELLPsyndrome was defined when all laboratory parameters were present and incomplete when one or more but not all laboratory findings were present. All patients had stable clinical conditions and were evaluated with magnetic resonance of the liver and the main findings were recorded.

RESULTS. Average maternal age was $26.8 \pm 6.4$ years and gestational age at delivery was $34 \pm 26.8$ weeks. The MR imaging was performed between eight and 96 hours after diagnosis of HELLP syndrome (56 \pm 31 h). The most frequentfindings were ascitis in $20 \%(n=8)$, pleural effusion in 17.5\% and hepatic steatosis in 7.5\%. The periportal intensity signal was normal in all cases. Cases of liver infarction and sub-capsular or parenchymatous hematoma were notobserved.

CONCLUSION. Findings of magnetic resonance imaging of the liver in stable HELLP syndrome postpartum patients were few and unspecific. Severe liver injuries such as parenchymatous or sub-capsular hematoma, entailing life risk were not found. Results do not corroborate the use of magnetic resonance as routine examination for stable patients with HELLP syndrome. [Rev Assoc Med Bras 2008; 54(5): 436-4I]

KeY wORDS: HELLP syndrome. Pre-eclampsia. Magnetic resonance imaging. Pregnancy. High-risk. 


\section{REFERÊNCIAS}

I. Weistein L. Syndrome of hemolysis, elevated liver enzymes and low platelet count: a severe consequence of hypertension in pregnancy. Am J Obstet Gynecol. 1982; I 42: 1 59-67.

2. Sibai BM. The HELLP syndrome (hemolysis, elevated liver enzymes and low platelets): much about nothing? Am J Obstet Gynecol. 1990; 162:311-6.

3. Sibai BM, Ramadan MK, Usta I, Salamana M, Mercer BM, Friedman SA. Maternal morbity and mortality in 442 pregnancies with hemolysis, elevated liver enzymes, and low platelets (HELLP Syndrome). Am J Obstet Gynecol. 1993; 169:1000-6.

4. Amorim MMR, Katz L, Ávila MB, Araújo DE, Valença M, Albuquerque CJM, et al. Perfil das admissões em uma unidade de terapia intensiva obstétrica de uma maternidade brasileira. Rev Bras Saúde Matern Infant. 2006;6:55-62.

5. Katz L, Amorim MMR, Santos LC, Silva JLP. Síndrome HELLP: estratégias para acelerar a recuperação materna pós-parto. Femina. 2005;33:687-92.

6. Santos LC, Amorim MMR, Katz L, Albuquerque CJM. Terapia intensiva em obstetrícia. Rio de Janeiro: Medsi; 2004.

7. Aldemir M, Baç B, Taçyildiz I, Yagmur Y, Keles C. Spontaneous liver hematoma and a hepatic rupture in HELLP syndrome: report of two cases. Surg Today. 2002;32:450-3.

8. Audibert F, Friedman SA, Frangieh AY, Sibai BM. Clinical utility of strict diagnostic criteria for the HELLP (hemolysis, elevated liver enzymes, and low platelets) syndrome. Am J Obstet Gynecol. 1996; 175:460-4.

9. Barton JR, Sibai BM Diagnosis and management of hemolysis, elevated liver enzymes, and low platelets syndrome. Clin Perinatol. 2004:31:807-33.

I0. Martin JN Jr, Thigpen BD, Rose CH, Cushman J, Moore A, May WL. Maternal benefit of high-dose intravenous corticosteroid therapy for HELLP syndrome. Am J Obstet Gynecol. 2003; | 89:830-4.

I I. Martin JN Jr, Rose CH, Briery CM. Understanding and managing HELLP syndrome: the integral role of aggressive glucocorticoids for mother and child. Am J Obstet Gynecol. 2006; 195:9|4-34.

12. Doshi S, Zucker SD. Liver emergencies during pregnancy. Gastroenterol Clin North Am. 2003;32: I 213-27.

13. Magann EF, Perry KG, Chauhan SP, Graves GR, Blake PG, Martin JN. Neonatal salvage by week gestation in pregnancies complicated by HELLP syndrome. J Soc Gynecol Investig. 1994; I:206-9.

14. Curtin WM, Weinstein L. A review of HELLP Syndrome. J Perinatol. 1999; 19:138-43
15. Barton JR, Sibai BM. Hepatic imaging in HELLP Syndrome (hemolysis, elevated liver enzymes, and low platelet count). Am J Obstet Gynecol. 1996; 174: 1820-7.

16. Chiang KS, Athey PA, Lamki N. Massive hepatic necrosis in the HELLP Syndrome: CT correlation. J Comput Assist Tomogr. 199|; | 5:845-7.

17. Danrad R, Martin DR. MR Imaging of diffuse liver diseases. Magn Reson Imaging Clin N Am. 2005; 1 3:277-93.

18. Hamer OW, Aguirre DA, Casola G, Lavine JE, Woenckhaus M, Sirlin CB. Fatty lifer: Imaging patterns and pitfalls. Radiographics. 2006;26: I 637-53.

19. Hosono M, Togashi K, Kawakami S, Itoh K, Fukuoka M, Kobayashi F, et al. MR Demonstration of reversible periportal abnormal intensity in eclampsia. J Comput Assist Tomogr. 1994; 1 8: 1 43-5.

20. Siegelman ES, Outwater EK. MR Imaging Techniques of the Liver. Radiol Clin North Am. 1998:36:263-86.

21. Report of the National High Blood Pressure Education Program. Working group report on high blood pressure in pregnancy. Am J Obstet Gynecol. 2000; 183:SI-S22.

22. Krueger KJ, Hoffman BJ, Lee WM. Hepatic infarction associated with eclampsia. Am J Gastroenterol. 1990;85:588-92.

23. Egerman RS, Sibai BM. HELLP syndrome. Clin Obstet Gynecol. 1999;42:38I-9.

24. Schwartz RB, Feske SK, Polak JF, DeGirolami U, laia A, Beckner KM, et al. Preeclampsia-eclampsia: clinical and neuroradiographic correlates and insights into the pathogenesis of hypertensive encephalopathy. Radiology. 2000;217:37|-6.

25. Rahman TM, Wendon J. Severe hepatic dysfunction in pregnancy. Q J Med. 2002;95:343-57.

26. Zak IT, Dulai HS, Kish KK. Imaging of neurologic disorders associated with pregnancy and the postpartum period. Radiographics. 2007;27:95- 108

27. Sandhu BS, Sanyal AJ. Pregnancy and liver disease. Gastroenterol Clin North Am. 2003;32:407-36.

28. Magann EF, Martin JN. Twelve steps to optimal management of HELLP syndrome. Clin Obstet Gynecol. 1999;42:532-50.

Artigo recebido: 18/10/07 Aceito para publicação: 07/04/08 